\title{
THE CLOSED SOCLE OF AN AZUMAYA ALGEBRA
}

\author{
F. R. DEMEYER
}

\begin{abstract}
If $R$ is a Noetherian ring and $A$ is an Azumaya algebra over $R$ then an ideal $H(A)$ in $R$, called the closed socle of $A$, is defined and it is shown that $H(A)$ is independent of the representative $A$ in the Brauer group of $R$. When $R$ is a domain, the behavior of $H(A)$ under localization and passage to the quotient field is studied, and $H(A)$ is calculated when $R$ is the affine ring of a real curve.
\end{abstract}

Let $R$ denote a Noetherian, integrally closed domain and $A$ an Azumaya (central separable) algebra over $R$. In [6], D. Haile associated to $A$ an ideal in $R$ which he called the closed socle of $A$ and which we denote $H(A)$. In this note we show how to define the closed socle of an Azumaya algebra over any commutative Noetherian ring, give simplified proofs of results slightly more general than those in [6], and calculate the closed socle of an Azumaya algebra over the affine ring of a real curve. More specifically, if $R$ is a commutative Noetherian ring and $A$ is an Azumaya algebra over $R$ then $H(A)$ is defined and is independent of the choice of representative $A$ in its class in the Brauer group $B(R)$ of $R$. If $R$ is a local Noetherian domain with quotient field $F$ and maximal ideal $m$ and $\Sigma=A \otimes F$, then Index $(\Sigma) \geqslant \operatorname{Index}(A / m A)$ when $H(A)=R$. Thus, if $\Sigma=M_{n}(F)$ then $H(A)$ $=R$ if and only if $A=M_{n}(R)$. Also, if $A / m A$ is a division algebra then $A$ is a maximal order in a division algebra over $F$ when $H(A)=R$. A localization result is proved and consequences of these results for Noetherian domains are derived. If $R$ is the affine ring of a real curve $X$ and $A$ is an Azumaya algebra over $R$ then $H(A) \subseteq \Pi P_{x}$ where $P_{x}$ is the prime ideal in $R$ corresponding to a point $x \in X$ and $x$ runs over the singular points $x \in X$ which are isolated in the strong topology and for which $A / P_{x} A$ is not in the trivial class of $B\left(R / P_{x}\right)$. Throughout all unexplained terminology and notation is as in [4], and $\otimes$ always means $\otimes_{R}$.

1. We begin by extending the definition of the closed socle given in [6]. Let $R$ be a domain with quotient field $F$, let $A$ be an Azumaya algebra over $R$, and let $\Sigma=A \otimes F=A \cdot F$. A left ideal $L$ in $A$ is pure over $R$ in case $r a \in L$ for $0 \neq r \in R$ and $a \in A$ implies $a \in L$ ([2, p.199]). It is easy to show that there is a one-to-one order preserving correspondence between the $R$-pure left ideals $L$ in $A$ and the left ideals $L^{1}$ in $\Sigma$ by $L \rightarrow L \cdot F$ and $L^{1} \rightarrow L^{1} \cap A$. It follows that minimal pure left ideals exist in $A$. Let $I$ be the sum of the minimal $R$-pure left ideals in $A$, then the closed socle of $A$ is defined to be $I \cap R$ and is denoted $H(A)$. Observe that the set $I$ in $A$ is actually a two-sided ideal in $A$. It is clear that $I$ is a left ideal in $A$, and for $a \in A$ and minimal pure left ideal $L$ of $A$ either $L a=0$ or

Received by the editors September 20, 1978 and, in revised form, March 21, 1979.

AMS (MOS) subject classifications (1970). Primary 13A20; Secondary 14H99, 16A16. 
$(L a) F=(L F) a$ is a minimal left ideal of $\Sigma$, since $L a \subseteq(L F) a \cap A$ and ( $L F) a \cap A$ is a two-sided ideal in $A$. By the one-to-one correspondence between two-sided ideals of $A$ and $R([4,3.7$, p. 54]) it follows that $I=A \cdot H(A)$. This corresponds to the construction of the closed socle for normal domains given in [6]. Now assume $R$ is a reduced Noetherian ring. Then $(0)=\cap_{i=1}^{n} P_{i}$, where the $P_{i}$ are a uniquely determined set of prime ideals so that the intersection is irredundant. If $A$ is an Azumaya algebra over $R$ let $A_{i}=R / P_{i} \otimes A$, then $A_{i}$ is an Azumaya algebra over the domain $R / P_{i}$. From the above $H\left(A_{i}\right)$ is an ideal in $R / P_{i}$ whose natural inverse image is an ideal $I_{i}$ in $R$. We define the closed socle $H(A)$ of $A$ to be $\cap_{i=1}^{n} I_{i}$. If $R$ is just a commutative Noetherian ring let $N$ be the nil radical of $R$, and let $A$ be an Azumaya algebra over $R$. Above we defined $H(A / N A)$ in $R / N$. We define the closed socle $H(A)$ of $A$ to be the natural inverse image of $H(A / N A)$ in $R$. The calculation of the closed socle of an Azumaya algebra over the affine ring of a real curve given later illustrates the naturalness of this definition.

Lemma 1. Let $R$ be a domain and $P$ a prime ideal in $R$. Let $A$ be an Azumaya algebra over $R$, then $H\left(R_{P} \otimes A\right)=R_{P} \otimes H(A)$ where $R_{P}$ denotes the localization of $R$ at $P$.

Proof. One can check that there is a one-to-one correspondence between the minimal pure left ideals $L$ of $A$ and the minimal pure left ideals $L^{1}$ of $R_{P} \otimes A=$ $A R_{P}$ by $L \rightarrow L R_{P}$ and $L^{1} \rightarrow A \cap L^{1}$. Let $I$ be the sum of the minimal pure left ideals of $A$ and $I^{1}$ the sum of the minimal pure left ideals in $A^{1}$. Then $I \cdot R_{P}=$ $\Sigma L R_{P}$ where $L$ runs through the minimal pure left ideals in $A$. Thus $I R_{P}=I^{1}$. Now one can check that $H(A) R_{P}=H\left(R_{P} \otimes A\right)$.

We note here that if $S$ is a commutative $R$-algebra and $A$ is an Azumaya $R$-algebra it may not be the case that $H(S \otimes A)=S \otimes H(A)$. For example let $\mathbf{R}$ denote the field of real numbers, let $R=\mathbf{R}[x, y] /\left(x^{2}+y^{2}\right)$ and let $S$ be the integral closure of $R$. We later show that if $A$ is the Azumaya algebra generated by elements $i, j$ subject to $i^{2}=j^{2}=-1$ and $i j=-j i$ and $P$ is the maximal ideal in $R$ generated by $\{x, y\}$ then $H(A) \subseteq P$; but $H(S \otimes A)=S$ so $S \otimes H(A) \neq$ $H(S \otimes A)$.

LEMma 2. Let $A$ and $A^{1}$ be two Azumaya algebras in the same class of the Brauer group of the Noetherian ring $R$, then $H(A)=H\left(A^{1}\right)$.

Proof. Assume $R$ is a local domain. Then there is an Azumaya $R$-algebra $D$ with no idempotents other than 0 and 1 and positive integers $n$ and $k$ with $A \cong M_{n}(D), A^{1}=M_{k}(D)$ by Corollary 1 of [3]. In this case it suffices to show $H(D)=H\left(M_{n}(D)\right)$. Let $F$ be the quotient field of $R$ and $\Sigma=F \otimes D=F \cdot D$. Then $F \otimes M_{n}(D)=M_{n}(\Sigma)$. Let $e_{i j}$ be the matrix in $M_{n}(\Sigma)$ with a 1 in the $i, j$ entry and 0 elsewhere. Let $L$ be a minimal pure left ideal in $D$ so $L^{1}=\Sigma L$ is a minimal left ideal in $\Sigma$. Then $M_{n}(L)=\bigoplus_{i=1}^{n} M_{n}(L) e_{i i}$ and $M_{n}(\Sigma) \cdot M_{n}(L)=M_{n}(\Sigma \cdot L)=$ $\bigoplus_{i=1}^{n} M_{n}(\Sigma L) e_{i i}$. Moreover, since $L$ is $R$-pure in $\Sigma, M_{n}(L) e_{i i}$ is $R$-pure in $M_{n}(\Sigma)$ so $M_{n}(\Sigma L) e_{i i} \cap M_{n}(D)=M_{n}(L) e_{i i}$. Finally, $M_{n}(\Sigma L) e_{i i}$ is a minimal left ideal in $M_{n}(\Sigma)$ since $\Sigma \cdot L$ is a minimal left ideal in $\Sigma$. Thus 


$$
\begin{aligned}
M_{n}(L) & =M_{n}(\Sigma L) \cap M_{n}(D)=\left(\bigoplus_{i=1}^{n} M_{n}(\Sigma L) e_{i i}\right) \cap M_{n}(D) \\
& \supseteq \bigoplus_{i=1}^{n} M_{n}(\Sigma L) e_{i i} \cap M_{n}(D)=\bigoplus_{i=1}^{n} M_{n}(L) e_{i i}=M_{n}(L) .
\end{aligned}
$$

Thus $M_{n}(L)$ is contained in the sum of the minimal pure left ideals in $M_{n}(D)$ so $H(D) \subseteq H\left(M_{n}(D)\right)$. For the reverse inclusion let $I$ be the sum of the minimal pure left ideals $L$ of $D$. Let $J^{\prime}$ be the sum of minimal pure left ideals in $M_{n}(D)$. By 3.5, p. 22 in [4] we know $J^{\prime}=M_{n}(J)$ for some two-sided ideal $J$ in $D$. We have already shown $I \subseteq J$. Let $\left(x_{i j}\right)$ be an $n \times n$ matrix in $M_{n}(D)$ with $\left(x_{i j}\right) \notin M_{n}(I)$ yet $\left(x_{i j}\right) \in L^{1}$ for some minimal pure left ideal $L^{1}$ of $M_{n}(D)$. If $x_{i j} \notin I$ then $\left(e_{i i}\right)\left(x_{i j}\right)\left(e_{j j}\right)$ is contained in $M_{n}(D)$ and in the minimal left ideal $L^{1} e_{i j}$. Thus if $X_{i j}$ is the matrix whose $i j$ th entry is $x_{i j}$ and all others are 0 then $X_{i j} \in L^{1} e_{j j} \cap M_{n}(D)$ is in a minimal pure left ideal in $M_{n}(D)$, so $M_{n}(\Sigma) X_{i j}$ is a minimal left ideal in $M_{n}(\Sigma)$. Thus $\Sigma x_{i j}$ must be a minimal left ideal in $\Sigma$. Thus $x_{i j} \in \Sigma x_{i j} \cap D \subseteq I$ which contradicts the choice of $x_{i j}$.

Now let $R$ be any commutative Noetherian ring with nil radical $N$. If $A$ is equivalent to $A^{1}$ in $B(R)$ then $A / N A$ is equivalent to $A^{1} / N^{1}$ in $B(R / N)$ so it suffices to show $H(A / N(A))=H\left(A^{1} / N A^{1}\right)$. Similarly, we can assume $R$ is a domain for if we write $(0)$ as the irredundant intersection of a unique set $\left\{P_{i}\right\}_{i=1}^{n}$ of prime ideals and if $H\left(A / P_{i} A\right)=H\left(A^{1} / P_{i} A^{1}\right)$ for all $i$ then $H(A)=H\left(A^{1}\right)$. If $R$ is a Noetherian domain and $P$ is any prime ideal in $R$ we know $R_{P} \otimes H(A)=R_{P} \otimes$ $H\left(A^{1}\right)$ by the first part of the proof since $R_{P} \otimes A$ is equivalent to $R_{P} \otimes A^{1}$ in $B\left(R_{P}\right)$. Thus if $H(A)=\cap{ }_{i=1}^{n} Q_{i}^{n_{i}}$ and $H\left(A^{1}\right)=\bigcap_{i=1}^{n} Q_{i}^{m_{i}}$ where $\left\{Q_{i}\right\}$ is an irredundant collection of prime ideals and $m_{i}, n_{i} \geqslant 0$ then $m_{i}=n_{i}$ for all $i$ so we always have $H(A)=H\left(A^{1}\right)$.

If $A$ is an Azumaya algebra over a field $F$ then the index of $A$ is the square root of the dimension of the division algebra part of $A$ over $F$. The next result is a generalization (with an easier proof) of Theorem 4.6 of [6].

THEOREM 1. Let $R$ be a local Noetherian domain with maximal ideal $m$ and quotient field $F$. Let $A$ be an Azumaya algebra over $R$ and $\Sigma=F \otimes A$. If $H(A)=R$ then Index $\Sigma \geqslant \operatorname{Index} A / m A$. Moreover, if Index $\Sigma=\operatorname{Index} A / m A$ then $A=M_{n}(B)$ where $B$ is an Azumaya $R$-algebra such that $F \otimes B$ is a division algebra.

Proof. Assume $H(A)=R$. Then there is a minimal pure left ideal $L$ of $A$ with $L \& m A$. Let $A_{0}=\Sigma L a$ where the sum runs over those $a \in A$ such that $L a \notin m A$. Then $A_{0}$ is an $R$-submodule of $A$ and $\left(A_{0}+m A\right) / m A$ is a two-sided ideal in $A / m A$. Since $A / m A$ is simple, $\left(A_{0}+m A\right) / m A=A / m A$, so by Nakayama's lemma, $A_{0}=A$. Therefore $A=\Sigma L a$ where the sum is taken over finitely many $a \in A$ such that $L a \& m A$. Let $\overline{L a}=F \cdot L a$. Then $\Sigma=\overline{L a_{1}} \oplus \cdots \oplus \overline{L a_{n}}$. Thus $A \supseteq L a_{1} \oplus \cdots \oplus L a_{n}=A_{1}$ and each $L a_{i} \& m A_{i}$ and $A_{1}$ is an $R$-submodule of $A$. Note that $\left(A_{1}+m A\right) / m A=\left(L a_{1}+m A\right) / m A \oplus \cdots \oplus\left(L a_{n}+m A\right) / m A$ and each summand on the right is a nonzero ideal in $A / m A$. This proves Index $\Sigma>$ Index $A / m A$. If Index $\Sigma=\operatorname{Index} A / m A$ then $\left(A_{1}+m A\right) / m A=A / m A$ so by 
Nakayama's lemma $A_{1}=A$. Let $B^{0}=\operatorname{Hom}_{A}\left(L a_{1}, L a_{1}\right)$. Then $B$ is an Azumaya algebra over $R$ and $A=\operatorname{Hom}_{B^{0}}\left(L a_{1}, L a_{1}\right)=M_{n}(B)$, with $B$ a maximal order in the division algebra component of $\Sigma$.

Corollary 1. Let $R$ be a local Noetherian domain with quotient field $F$, and let $A$ be an Azumaya $R$-algebra. If $F \otimes A \cong M_{n}(F)$ then $A \cong M_{n}(R)$ if and only if $H(A)=R$.

Proof. If $A \cong M_{n}(R)$ then it is easy to see that $H(A)=R$ (this also follows from Lemma 2). Conversely, Index $F \otimes A=1 \geqslant \operatorname{Index} A / m A$ so the inequality is an equality and the result follows from Theorem 1 .

Corollary 2. Let $R$ be a Noetherian domain with field of quotients $F$. Suppose $A$ is an Azumaya $R$-algebra with $F \otimes A \cong M_{n}(F)$. Let $P$ be a prime ideal in $R$, then $R_{P} \otimes A \cong M_{n}\left(R_{P}\right)$ if and only if $H(A) \subseteq P$.

Proof. Combine Lemma 1 and Corollary 1.

Corollary 3. Let $R$ and $A$ be as in Theorem 1. If $A / m A$ is a division algebra and $H(A)=R$ then $F \otimes A$ is a division algebra.

Proof. By Theorem 1, Index $F \otimes A \geqslant \operatorname{Index} A / m A$ since [Index $A / m A]^{2}=$ $\operatorname{Rank}_{R / m}(A / m A)=\operatorname{Rank}_{R}(A)=\operatorname{Rank}_{F}(F \otimes A) \geqslant[\operatorname{Index} F \otimes A]^{2}$. Thus [Index $F \otimes A]^{2}=\operatorname{Rank}_{F}(A)$ and $F \otimes A$ is a division algebra.

THEOREM 2. Let $R$ be the affine ring of a real curve $X$. For each closed point $x \in X$ let $P_{x}$ be the corresponding maximal ideal in $R$. Let $A$ be an Azumaya $R$-algebra. Then the longest product of ideals $P_{x}$ containing $H(A)$ has factors $P_{x}$ satisfying all of the following.

1. $x$ is a real singular point on $X$.

2. $x$ is isolated in the strong topology on the real points of the irreducible component $X_{i}$ of $X$ containing $x$.

3. $A / P_{x} A$ does not represent the trivial class in $B\left(R / P_{x}\right)$.

Proof. For each $x \in X$ let $R(x)=R / P_{x}$ and $A(x)=A / P_{x} A$. Since $X$ is a real curve $R(x)$ is either the real or complex numbers. If $R(x)$ is the real number $\mathbf{R}$ then either $A(x)$ is a matrix algebra over $\mathbf{R}$ or $A(x)$ is a matrix algebra over the division algebra of real quaternions. In [5] the Azumaya algebras $A$ over $R$ are characterized as continuously parameterized systems of Azumaya algebras $A(x)$ over the real points $x \in X$ with the strong topology. If $X$ is irreducible then $R$ is a domain and the quotient field $F$ of $R$ is the field of rational functions on $X$. By Remark 3.4 of [5] the real points $x$ for which $A(x)$ is a matrix algebra over the quaternions, yet $F \otimes_{R(x)} A(x)$ is a matrix algebra over $F$, are precisely the real singular points $x \in X$ which are isolated in the strong topology and for which $A(x)$ is a matrix algebra over the quaternions. Combining Theorem 1 and Lemma 1 we see that $A(x)$ is a matrix algebra over the quaternions yet $F \otimes A(x)$ is a matrix algebra over $F$ if and only if $P_{x}$ contains $H(A)$. If $X$ is reducible we can assume $R$ is reduced. Let $(0)=\cap{ }_{i=1}^{n} P_{i}$ where $\left\{P_{i}\right\}$ is an irredundant set of prime ideals of $R$. The rings $R / P_{i}$ are the affine rings of the irreducible components $X_{i}$ of $X$. If 
$A_{i}=R / P_{i} \otimes A$ then $H\left(A_{i}\right)$ is contained in the product of the maximal ideals which correspond to the real singular points $x_{i}$ on $X_{i}$ which are isolated in the strong topology on $X_{i}$ and for which $A(x)$ is a matrix algebra over the quaternion algebra. This completes the proof of the theorem.

\section{REFERENCES}

1. M. Auslander and O. Goldman, The Brauer group of a commutative ring, Trans. Amer. Math. Soc. 97 (1960), 367-409.

2. C. W. Curtis and I. Reiner, Representation theory of finite groups and associative algebras, Interscience, New York, 1962.

3. F. R. DeMeyer, Projective modules over central separable algebras, Canad. J. Math. 21 (1969), 39-43.

4. F. R. DeMeyer and E. Ingraham, Separable algebras over commutative rings, Lecture Notes in Math., vol. 181, Springer-Verlag, Berlin and New York, 1971.

5. F. R. DeMeyer and M. A. Knus, The Brauer group of a real curve, Proc. Amer. Math. Soc. 58 (1976), 227-232.

6. D. Haile, The closed socle of a central separable algebra, J. Algebra 51 (1978), 97-106.

Department of Mathematics, Colorado State University, Fort Colnns, Colorado 80523 